\title{
An unusual cause of tonsillar asymmetry
}

\author{
D Karamchandani, SK El-Shunnar, JM Fussey, SF Ahsan, S Bhatia
}

Shrewsbury and Telford Hospital NHS Trust, UK

ABSTRACT

We present the first reported case of tonsillar asymmetry secondary to a mandibular osteoma. Computed tomography rather than tonsillectomy for histology prevented the distress and risk of unnecessary surgery, and the patient was managed conservatively.

\section{KEYWORDS}

Palatine tonsil - Tonsillectomy - Head and neck neoplasm - Osteoma

Accepted 3 January 2017

CORRESPONDENCE TO

Jonathan Fussey, E: jfussey@doctors.org.uk

Although often the result of a benign process, unilateral palatine tonsillar hypertrophy is usually treated with tonsillectomy owing to the suspicion of underlying malignancy such as lymphoma or carcinoma. ${ }^{1}$ The suspicion of malignancy increases in older patients who are symptomatic. A comprehensive literature search using the terms tonsil, mandible and osteoma did not reveal any reported cases of mandibular osteoma presenting as tonsillar asymmetry. We feel that the case reported below has important implications for clinical practice.

\section{Case history}

A 76-year-old Caucasian woman presented with a 3-month history of a right-sided sore throat. She was referred to the head and neck clinic as an urgent two-week referral with suspicion of a tonsillar malignancy. She had initially been treated with antibiotics, which did not relieve her symptoms. She reported no dysphagia, hoarseness or weight loss. She had a past medical history of diverticulitis but was otherwise fit and well with no known allergies and no regular medications. She was a non-smoker with low alcohol consumption. Oropharyngeal examination revealed a very large nonulcerated right tonsil (Fig 1). Palpation of the neck was unremarkable, as was flexible nasolaryngoscopy. The tonsil was found to be firm and tender on palpation. Given her age and the associated tenderness on palpation, computed tomography (CT) of her head and neck was organised prior to scheduling her for panendoscopy and tonsillectomy. Biochemical and haematological investigations were normal.

The CT revealed a large osteoma arising from the ramus of the mandible on the right (Fig 2). This was found to be displacing the right tonsil medially, with no abnormality in the tonsil itself and no cervical lymphadenopathy. The patient was largely asymptomatic apart from mild discomfort in the throat. She was subsequently reviewed by the maxillofacial team, who advised conservative management with ongoing follow-up visits.

\section{Discussion}

Tonsillectomy for unilateral tonsillar enlargement should be considered very carefully as there are clear risks with tonsillectomy (eg primary and secondary post-tonsillectomy haemorrhage) and also complications associated with general anaesthesia. ${ }^{1}$ The index of suspicion of a malignant process should be high in older age groups, especially if there is a history of tobacco and alcohol consumption or a past history of malignancy. ${ }^{2,3}$ There is debate over whether a "watch and wait' approach can be adopted for unilateral tonsillar enlargement to avoid unnecessary surgery and its complications.

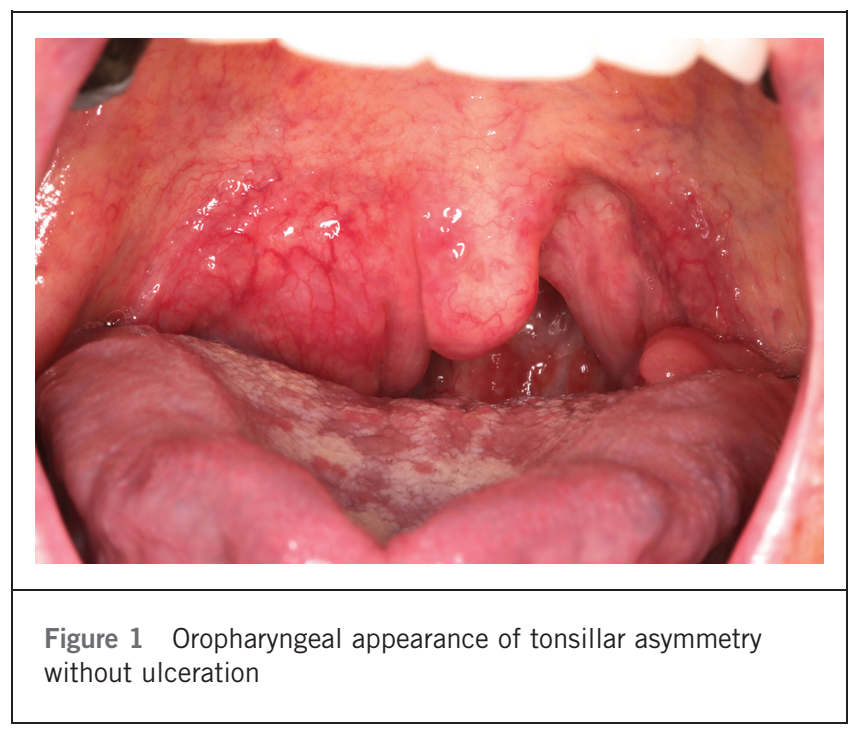




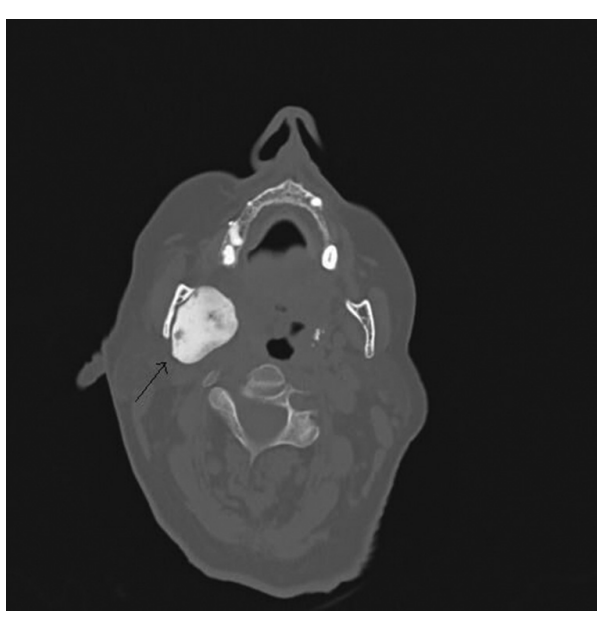

Figure 2 Axial computed tomography showing right tonsil medialisation by osteoma (arrow)

In our case, proceeding to a tonsillectomy without imaging could have led to multiple complications owing to the displacement of the tonsil, as can be seen in the CT (Fig 2). The dissection of the tonsil would have been likely to be difficult and may have led to significant bleeding. Preoperative CT in this situation prevented unnecessary surgery and morbidity.

Osteomas are found mainly in the craniofacial bones. An osteoma is a benign osteogenic tumour characterised by compact or cancellous bone proliferation. It may be classified as peripheral, central or extraskeletal. Peripheral osteomas arise from the periosteum, central osteomas from the endosteum and extraskeletal osteomas in the soft tissue. ${ }^{4}$ The most frequent sites affected in the mandible are the lingual surface of the body (often bilaterally), followed by the condyle, angle, ascending ramus, coronoid process, anterior body and sigmoid notch. ${ }^{5}$ Osteomas can occur at any age, and both sexes are affected equally. ${ }^{6}$ As in our patient, they are usually slow growing and largely asymptomatic.

\section{Conclusions}

The possibility of an underlying malignancy should be considered in unilateral tonsillar hypertrophy and each case should be managed on an individual basis. However, one must remember that the tonsils can appear hypertrophic owing to medialisation (such as from deep lobe of parotid tumours). In such circumstances, the tonsils should be palpated, and if the tonsil is found to be tender and firm, then appropriate imaging should be considered prior to surgical intervention. As demonstrated in our case, CT may prevent unnecessary tonsillectomy and its associated morbidity.

\section{References}

1. Spinou C, Kubba, H, Konstantinidis I, Johnston A. Role of tonsillectomy in histology for adults with unilateral tonsillar enlargement. Br J Oral Maxillofac Surg 2005; 43: 144-147.

2. Tobias Gómez $\mathrm{S}$, Palomar Asenjo V, Borràs Perera $\mathrm{M}$ et al. Clinical significance of unilateral tonsillar enlargement. Acta Otorrinolaringol Esp 2009; 60: 194-198.

3. Sunkaraneni VS, Jones SE, Prasai A, Fish BM. Is unilateral tonsillar enlargement alone an indication for tonsillectomy? J Laryngol Otol 2006; 120: E21.

4. Kaplan I, Calderon S, Buchner A. Peripheral osteoma of the mandible: a study of 10 new cases and analysis of the literature. J Oral Maxillofac Surg 1994; 52: 467-470.

5. Sayan NB, Uçok C, Karasu HA, Günhan O. Peripheral osteoma of the oral and maxillofacial region: a study of 35 new cases. J Oral Maxillofac Surg 2002; 60: 1,299-1,301.

6. Kaplan I, Nicolaou Z, Hatuel D, Calderon S. Solitary central osteoma of the jaws: a diagnostic dilemma. Oral Surg Oral Med Oral Pathol Oral Radiol Endod 2008; 106: e22-e29. 